\title{
The correlation of the steady-state gas/water relative permeabilities of porous media with gas and water capillary numbers
}

\author{
Christos D. Tsakiroglou* \\ Foundation for Research and Technology Hellas - Institute of Chemical Engineering Sciences, Stadiou Street, \\ Platani, 26504 Patras, Greece
}

Received: 2 February 2018 / Accepted: 18 March 2019

\begin{abstract}
The steady-state gas, $k_{\mathrm{rg}}$, and water, $k_{\mathrm{rw}}$, relative permeabilities are measured with experiments of the simultaneous flow, at varying flow rates, of nitrogen and brine (aqueous solution of $\mathrm{NaCl}$ brine) on a homogeneous sand column. Two differential pressure transducers are used to measure the pressure drop across each phase, and six ring electrodes are used to measure the electrical resistance across five segments of the sand column. The electrical resistances are converted to water saturations with the aid of the Archie equation for resistivity index. Both $k_{\mathrm{rw}}$ and $k_{\mathrm{rg}}$ are regarded as power functions of water, $\mathrm{Ca}_{\mathrm{w}}$, and gas, $\mathrm{Ca}_{\mathrm{g}}$, capillary numbers, the exponents of which are estimated with non-linear fitting to the experimental datasets. An analogous power law is used to express water saturation as a function of $\mathrm{Ca}_{\mathrm{w}}$, and $\mathrm{Ca}_{\mathrm{g}}$. In agreement to earlier studies, it seems that the two-phase flow regime is dominated by connected pathway flow and disconnected ganglia dynamics for the wetting fluid (brine), and only disconnected ganglia dynamics for the non-wetting fluid (gas). The water saturation is insensitive to changes of water and gas capillary numbers. Each relative permeability is affected by both water and gas capillary numbers, with the water relative permeability being a strong function of water capillary number and gas relative permeability depending strongly on the gas capillary number. The slope of the water relative permeability curve for a gas/water system is much higher than that of an oil/water system, and the slope of the gas relative permeability is lower than that of an oil/water system.
\end{abstract}

\section{Introduction}

The relative permeability functions of reservoir rocks are fed as input data in macroscopic simulators of enhanced oil and gas recovery processes, and affect the numerical predictions of the spatio-temporal evolution of hydrocarbon saturation, and implicitly, the design of strategies for reservoir exploitation (Kamali and Hussain, 2017; Sherafati and Jessen, 2017).

The relative permeability functions of porous rocks for a specific fluid system (gas/water, gas/oil, oil/water, gas/oil/ water) are commonly measured with special core analysis tests performed on representative rock samples under transient (Sidiq et al., 2017) or steady-state (Reynolds and Krevor, 2015) conditions. Alternatively, computational methods such as the Lattice-Boltzman technique (Ramstad et al., 2012; Zhang et al., 2016), the pore network modeling (Ramstad and Hansen, 2006; Grøva and Hansen, 2011), and the tube bundle model (Wu et al., 2016) might be used to calculate the relative permeabilities from information concerning the pore space morphology, wettability, and

\footnotetext{
* Corresponding author: ctsakir@iceht.forth.gr
}

fluid dynamics. The steady-state two-phase flow is established by injecting two fluids through a porous medium at fixed flow rates, and has extensively be analyzed by using hierarchical simulations at pore- and network-scale (Constantinides and Payatakes, 1996; Ramstad and Hansen, 2006; Sinha and Hansen, 2012; Valavanides, 2012; Valavanides et al., 2016), and systematic experimental approaches in model porous media (Avraam and Payatakes, 1995a, 1995b, 1999; Tsakiroglou et al., 2007; Gutierrez et al., 2008; Tallakstad et al., 2009a, 2009b; Erpelding et al., 2013; Tsakiroglou et al., 2015). One of the main conclusions is that the wetting fluid (water) retains its connectivity along its flow path while the nonwetting fluid (oil or gas) may move either as a connected pathway or as a population of disconnected ganglia which undergo dynamic breakup and coalescence (Avraam and Payatakes, 1995a; Valavanides et al., 1998; Tallakstad et al., 2009a, 2009b). Ganglion dynamics may contribute to the understanding of capillary trapping for supercritical $\mathrm{CO}_{2}$ under reservoir conditions (Wang et al., 2013).

In the present work, experiments of the steady-state flow of nitrogen/brine (NaCI solution) through a sand column equipped with electrodes are used to determine the gas/water relative permeabilities, and water saturation, 
and quantify their correlations with the current flow rates, in terms of power laws. Such information might be helpful for a variety of practical applications: the fate of $\mathrm{CO}_{2}$ ganglia during its storage in deep aquifers or depleted oil reservoirs (Terzi et al., 2014); the enhanced oil recovery by foams (Eftekhari and Farajzadeh, 2017) or WaterAlternating-Gas (WAG) (Sherafati and Jessen, 2017); the exploitation of methane hydrates (Johnson et al., 2011).

\section{Materials and methodology}

\subsection{Experimental setup}

Experiments of the steady-state and simultaneous flow of an aqueous and a gas phase were performed on a long horizontal column of PVC which was filled with well-sorted sand (Tab. 1) and equipped with six ring electrodes and an in-house constructed multi-point resistivity meter for the measurement of the electrical conductivity across various segments of the column (Fig. 1; Aggelopoulos and Tsakiroglou, 2008). All measuring devices were connected with the data acquisition card of a host computer. The apparatus was placed inside a thermostatted chamber to keep the temperature constant and equal to $25^{\circ} \mathrm{C}$ and avoid any temperature-induced disturbances of the electrical conductance. The multiple-point resistivity meter allowed the simultaneous measurement of the electrical resistance across various segments of the column with respect to a reference electrode. The total pressure drop across each phase was measured with pressure transducers connected to the inlet tubes (Fig. 2).

Initially, the sand column was evacuated and filled with brine $\left(\mathrm{NaCl}\right.$ aqueous solution of concentration, $C_{\mathrm{NaCl}}=$ $20 \mathrm{~g} / \mathrm{L}$ ) by capillary imbibition. Then, gas nitrogen was injected at constant flow rate, $q_{\mathrm{g}}=10 \mathrm{~mL} / \mathrm{min}$, with the aid of a gas mass flow controller, until reaching an irreducible wetting phase saturation. Afterwards, simultaneously with $\mathrm{N}_{2}$, brine started to be injected at a flow rate $0.2 \mathrm{~mL} / \mathrm{min}$ (Fig. 1). Steady-state conditions were achieved when the time-averaged values of gas and brine inlet pressures along with the measured electrical conductances were stabilized. The gas flow rate was kept constant and the brine flow rate was increased stepwise from 0.2 to $5 \mathrm{~mL} / \mathrm{min}$ by successive steps (Fig. 3). At each step, the steady-state space-averaged relative permeabilities were calculated for the gas, $\left\langle k_{\mathrm{rg}}\right\rangle$, and aqueous, $\left\langle k_{\mathrm{rw}}\right\rangle$, phase by using the Darcy law and the time-averaged pressure drop across each phase, respectively

$$
\begin{gathered}
u_{\mathrm{w}}=\frac{q_{\mathrm{w}}}{A}=\left(\frac{k\left\langle k_{\mathrm{rw}}\right\rangle}{\mu_{\mathrm{w}}}\right)\left(\frac{\Delta P_{\mathrm{w}}}{L}\right), \\
u_{\mathrm{g}}=\frac{q_{\mathrm{g}}}{A}=\left(\frac{k\left\langle k_{\mathrm{rg}}\right\rangle}{\mu_{\mathrm{g}}}\right)\left(\frac{\Delta P_{\mathrm{g}}}{L}\right)\left(\frac{P_{\mathrm{m}}}{P_{\mathrm{g}, \mathrm{in}}}\right),
\end{gathered}
$$

where $u_{\mathrm{w}}, u_{\mathrm{g}}$ are the water and gas superficial velocities; $A, L$ are the cross-sectional area and length of the porous medium; $\mu_{\mathrm{w}}, \mu_{\mathrm{g}}$ are the water and gas viscosities; $\Delta P_{\mathrm{w}}$, $\Delta P_{\mathrm{g}}$ are the pressure drops across the aqueous and gas
Table 1. Properties of sand column and fluids.

\begin{tabular}{lcc}
\hline Parameter & Symbol & Value \\
\hline Column length & $L$ & $0.3 \mathrm{~m}$ \\
Column diameter & $D$ & $0.05 \mathrm{~m}$ \\
Porosity & $\varphi$ & 0.42 \\
Average grain diameter* & $d_{\mathrm{g}}$ & $\sim 188 \mu \mathrm{m}$ \\
Average pore throat diameter** & $d_{\mathrm{p}}$ & $\sim 28 \mu \mathrm{m}$ \\
Absolute permeability & $k$ & $25 \times 10^{-12} \mathrm{~m}^{2}$ \\
Aqueous phase viscosity & $\mu_{\mathrm{w}}$ & $0.95 \times 10^{-3} \mathrm{~Pa} \mathrm{~s}^{-}$ \\
Gas phase viscosity & $\mu_{\mathrm{w}}$ & $1.8 \times 10^{-5} \mathrm{~Pa} \mathrm{~s}$ \\
Viscosity ratio & $\kappa$ & $\sim 0.02$ \\
Interfacial tension & $\gamma_{\mathrm{wg}}$ & $72 \times 10^{-3} \mathrm{~N} \mathrm{~m}^{-1}$ \\
\hline
\end{tabular}

${ }^{*}$ It is considered equal to the mean value of the lower $(\sim 125 \mu \mathrm{m})$ and upper $(\sim 250 \mu \mathrm{m})$ limit of grain size range. ** It is considered equal to the diameter of the circle that is inscribed in the "window" left between three circles of equal diameter $d_{\mathrm{g}}$ and touching each other $\left(d_{\mathrm{p}}=0.15 d_{\mathrm{g}}\right)$.

phase; $P_{\mathrm{m}}=\left(P_{\mathrm{g}, \text { in }}+P_{\mathrm{g}, \text { out }}\right) / 2$ is the mean gas pressure and $P_{\mathrm{g}, \text { in }}, P_{\mathrm{g}, \text { out }}$ are the inlet and outlet gas pressures. Given that the range of gas pressures is quite low (gauge pressures $\sim 1-5 \mathrm{kPa}$ ), the effect of gas compressibility on measured absolute and relative permeability is negligible and the last term of the right-hand side of equation (1b) was ignored.

The brine flow rate was kept constant and the gas flow rate was reduced to $q_{\mathrm{g}}=5.0 \mathrm{~mL} / \mathrm{min}$ (Fig. 3). When steady-state two-phase flow conditions were established, the gas flow rate was kept constant and the brine flow rate started to decrease stepwise from 5.0 to $0.5 \mathrm{~mL} / \mathrm{min}$, so that, at each step, the steady-state relative permeability was determined for each phase (Fig. 3). The entire procedure was repeated for gas flow rates $q_{\mathrm{g}}=2.0,1.0$ and $0.5 \mathrm{~mL} / \mathrm{min}$ (Fig. 3). Totally, the gas and water relative permeability along with the water saturation were determined for 25 steady-states, associated with 25 pairs of gas and brine flow rates.

At the end of each steady-state step, the total mass of brine, collected in a storage tank connected to the outlet port (Fig. 2), was weighted and the water saturation averaged over the column, $\left\langle S_{\mathrm{w}}\right\rangle$, was estimated. The electrical resistivity index, $I_{\mathrm{R}}$, of a porous medium partially saturated with an electrolyte solution is defined as the ratio of the measured electrical resistance to the resistance that would have been measured if the porous medium had fully been saturated with the electrolyte solution of equal concentration. The measured, $I_{\mathrm{R}}-\left\langle S_{\mathrm{w}}\right\rangle$, datasets were fitted to the Archie law

$$
I_{\mathrm{R}}=\left\langle S_{\mathrm{w}}\right\rangle^{-n}
$$

to estimate the saturation exponent, $n$. The values of resistivity index, $\left(I_{\mathrm{R}}\right)_{i j}$, measured across each segment of the column were substituted in equation (2) to calculate the water saturation, $\left\langle S_{\mathrm{w}}\right\rangle_{i j}$, averaged over each column segment. It should be mentioned that the tests 


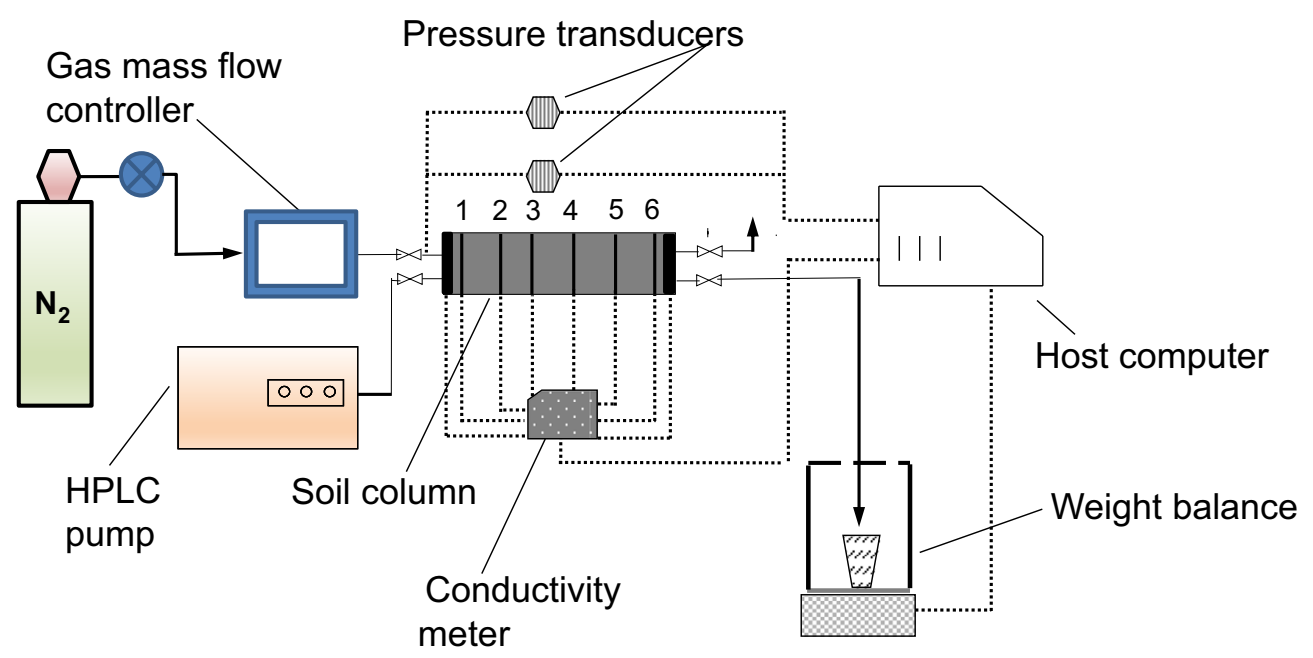

Fig. 1. Experimental apparatus of the steady-state two-phase flow in soil columns.

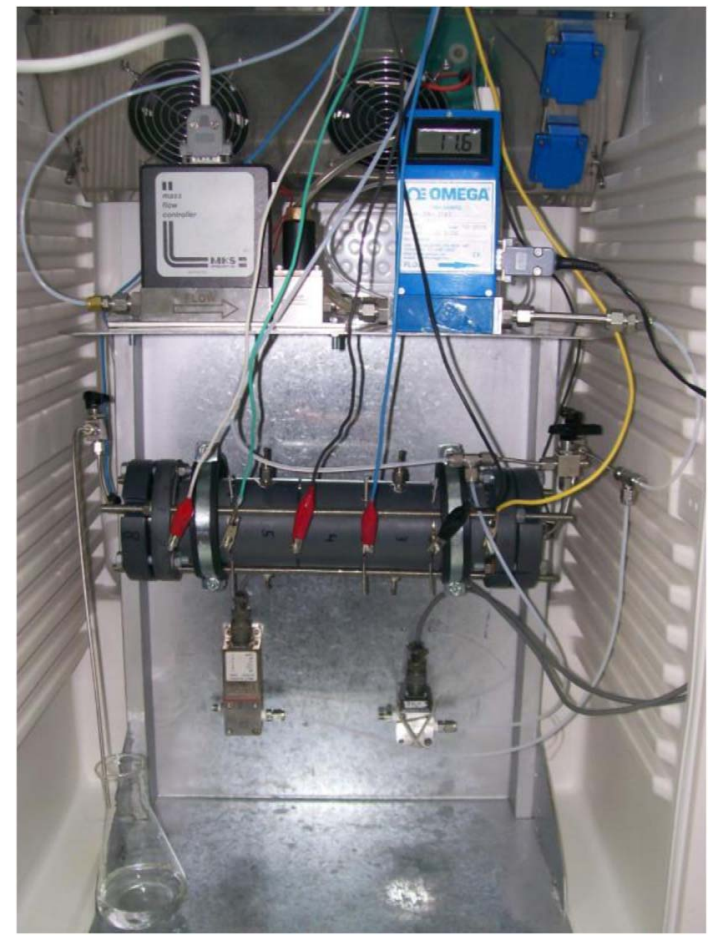

Fig. 2. Sand column equipped with two pressure transducers, a gas mass flow controller, and six electrodes.

of the simultaneous two-phase flow started from the initial condition of irreducible wetting phase saturation (drainage) of the porous medium by varying the flow rates in the stepwise manner shown in Figure 3. It is not clear if and how the saturation history, namely the succession of the fluid injection steps, might affect the various flow regimes and measured relative permeability curves. On the other hand, if the tests started from the initial condition of residual non-wetting (gas) phase saturation (imbibition), then it would be expected that the different spatial distribution of fluids might result in different flow regimes and relative permeability curves for the same capillary numbers. Notwithstanding, a systematic parametric analysis is required for addressing the aforementioned issues.

\subsection{Relative permeabilities and numerical fitting}

Usually, the measured relative permeabilities are expressed as functions of saturation by using Corey-type models, albeit they depend on a variety of parameters. Given that the same water saturation might be reached at two different pairs of flow-rates for the gas/water system, the corresponding relative permeabilities should be fitted with Corey-type models with the fitting parameters being functions of the flow rates (Tsakiroglou et al., 2015).

To avoid the abovementioned contradiction, we introduce a correlation where the water saturation and relative permeabilities are regarded as dependent variables. For each experiment, the water saturation, $\left\langle S_{\mathrm{w}}\right\rangle$, along with the gas, $\left\langle k_{\mathrm{rg}}\right\rangle$, and water, $\left\langle k_{\mathrm{rw}}\right\rangle$, relative permeability, measured as space-averaged variables across the entire column, are considered as functions of two independent variables: the capillary numbers of aqueous, $\mathrm{Ca}_{\mathrm{w}}$, and gas, $\mathrm{Ca}_{\mathrm{g}}$, phases, defined by

$$
\begin{gathered}
\mathrm{Ca}_{\mathrm{w}}=\mu_{\mathrm{w}} q_{\mathrm{w}} /\left(A \gamma_{\mathrm{gw}}\right), \\
\mathrm{Ca}_{\mathrm{g}}=\mu_{\mathrm{g}} q_{\mathrm{g}} /\left(A \gamma_{\mathrm{gw}}\right),
\end{gathered}
$$

where $\mu_{\mathrm{i}}=$ viscosity of phase $\mathrm{i}(\mathrm{i}=\mathrm{w}, \mathrm{g}) ; q_{\mathrm{i}}=$ flow rate of phase i $(\mathrm{i}=\mathrm{w}, \mathrm{g}) ; \gamma_{\mathrm{gw}}=$ interfacial tension; $A=$ cross-sectional area of porous medium. The dependence of $\left\langle k_{\mathrm{rw}}\right\rangle$, $\left\langle k_{\mathrm{rg}}\right\rangle,\left\langle S_{\mathrm{w}}\right\rangle$ on $\mathrm{Ca}_{\mathrm{w}}, \mathrm{Ca}_{\mathrm{g}}$, is described by power laws of the form (Tsakiroglou et al., 2015)

$$
\begin{aligned}
& \left\langle k_{\mathrm{rw}}\right\rangle=a_{\mathrm{w}} \mathrm{Ca}_{\mathrm{w}}{ }^{{ }{ }_{\mathrm{w}}} \mathrm{Ca}_{\mathrm{g}}{ }^{{ }_{\mathrm{g}}}, \\
& \left\langle k_{\mathrm{rg}}\right\rangle=a_{\mathrm{g}} \mathrm{Ca}_{\mathrm{w}}{ }^{{ }^{\mathrm{w}}} \mathrm{Ca}_{\mathrm{g}}{ }^{{ }^{\mathrm{g}}},
\end{aligned}
$$




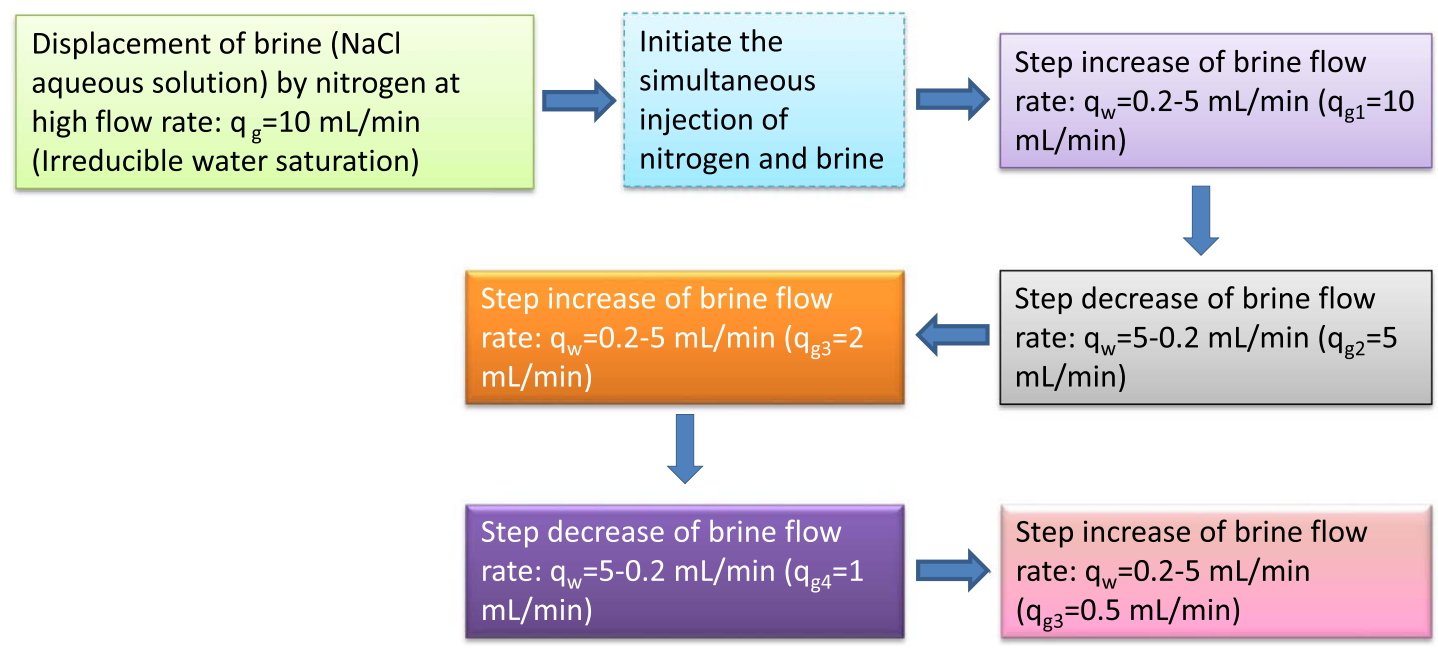

Fig. 3. Experimental procedure for the measurement of relative permeabilities for 25 pairs of flow rates for gas and water.

$$
\left\langle S_{\mathrm{w}}\right\rangle=a_{\mathrm{s}} \mathrm{Ca}_{\mathrm{w}}{ }^{c_{\mathrm{w}}} \mathrm{Ca}_{\mathrm{g}}{ }^{{ }^{\mathrm{g}}} \text {. }
$$

\section{Results and discussion}

\subsection{Resistivity index and water saturation profiles}

Initially, the Archie law, equation (2), was used to correlate the steady-state electrical resistivity across the entire column, $I_{\mathrm{R} 16}$, with the total water saturation, $S_{\mathrm{w} 16}$, calculated from mass balance of effluents. A saturation exponent $n=2.01$ with very satisfactory correlation coefficient was obtained (Fig. 4a). It is assumed that the steady-state values of resistivity index are also applicable to the transitional periods between successive steps, during which momentum transfer and immiscible displacement may take place (Tsakiroglou et al., 2015). Accounting for the negligible viscous resistance of the gas phase, this assumption is reasonable. Regarding the uncertainty of $S_{\mathrm{w}}$ measurements from $I_{\mathrm{R}}$ in accordance with Archie law, it should be reported that a variation in the value of resistivity index or saturation exponent $\sim 5 \%$ is reflected in a variation in the value of water saturation $\sim 2.5 \%$ or $3.5 \%$, respectively. From this point of view, the error embedded in the values of water saturation resulting from Archie law is acceptable.

It was observed that at high $\mathrm{Cag}_{\mathrm{g}}$ values (Fig. $4 \mathrm{~b}$ ), the resistivity index across various segments of the sand column is a clearly decreasing function of $\mathrm{Ca}_{\mathrm{w}}$ (Fig. $4 \mathrm{~b}$ ), and subsequently the corresponding water saturation is an increasing function of $\mathrm{Ca}_{\mathrm{w}}$. At low $\mathrm{Ca}_{\mathrm{g}}$ values, the resistivity index (and hence the water saturation) changes weakly with $\mathrm{Ca}_{\mathrm{w}}$ (Fig. 4c). Overall, a weak variation of water saturation with $\mathrm{Ca}_{\mathrm{w}}$ is expected.

The effect of the Bond number on the immiscible displacement pattern, resistivity index, and saturation exponent of a porous medium has been analyzed in detail with transient two-phase flow tests performed on the same sand column at varying orientations (Aggelopoulos et al., 2005). In the present work, all tests were conducted at horizontal orientation, and therefore eventual effects of Bond number on the two-phase flow regime are expected to be evident (if exist) at the scale of the column diameter, $D$. At this length scale, the Bond number is approximated by the relation

$$
\mathrm{Bo}=\frac{\Delta \rho g D d_{\mathrm{p}}}{4 \gamma_{\mathrm{wg}}},
$$

where $\Delta \rho$ is the density difference of the two fluids (brine and gas) and $\mathrm{g}$ is the acceleration of gravity. Supposing that $\Delta \rho=1000 \mathrm{~kg} / \mathrm{m}^{3}$ and $g=9.81 \mathrm{~m} / \mathrm{s}^{2}$ and using the data of Table 1 , we get $\mathrm{Bo}=0.048$, and therefore the effects of gravity forces on the two-phase flow regime, over the scale of the column diameter, can be neglected. However, if the sand column was placed in vertical position, then at the scale of column length, $L$, the Bo $\sim 0.3$, and gravity may start affecting the two-phase flow regime.

For any step change from a pair to another pair of flow rates, there is a transitional period during which the measured variables (pressures, resistivity index) change (Figs. 5a-5d). Steady-state is established when the measured fluid pressures are stabilized (Figs. 5a and 5c) and the resistivity index measured over the various column segments tends to be stabilized (Figs. 5b and 5d). In general, the period of time required to establish steady-state conditions decreases with the flow rates increasing, and on average, this period was $\sim 10 \mathrm{~min}$ (Fig. 5).

The exponents $b_{\mathrm{w}}, b_{\mathrm{g}}, e_{\mathrm{w}}, e_{\mathrm{g}}, c_{\mathrm{w}}, c_{\mathrm{g}}$, quantify the dependence of $\left\langle k_{\mathrm{rw}}\right\rangle,\left\langle k_{\mathrm{rg}}\right\rangle,\left\langle S_{\mathrm{w}}\right\rangle$ on the flow rates, viscosity ratio, porous medium properties, and wettability. For oil/water systems where the non-wetting to wetting fluid viscosity ratio, $\kappa$, ranges from 0.65 to 3.35 (Avraam and Payatakes, 1995a, 1995b, 1999; Tsakiroglou et al., 2007, 2015) the flow regime of Connected Pathway Flows (CPF) in oil phase becomes evident at low water saturations (associated with low $\mathrm{Ca}_{\mathrm{w}}$ values), high oil flow rates (namely high, $\mathrm{Ca}_{\mathrm{o}}$, values) and relatively high values of viscosity ratio, $\kappa$ (Avraam and Payatakes, 1995a, 1995b). In contrast, at higher water saturations (or high $\mathrm{Ca}_{\mathrm{w}}$ values), and lower 

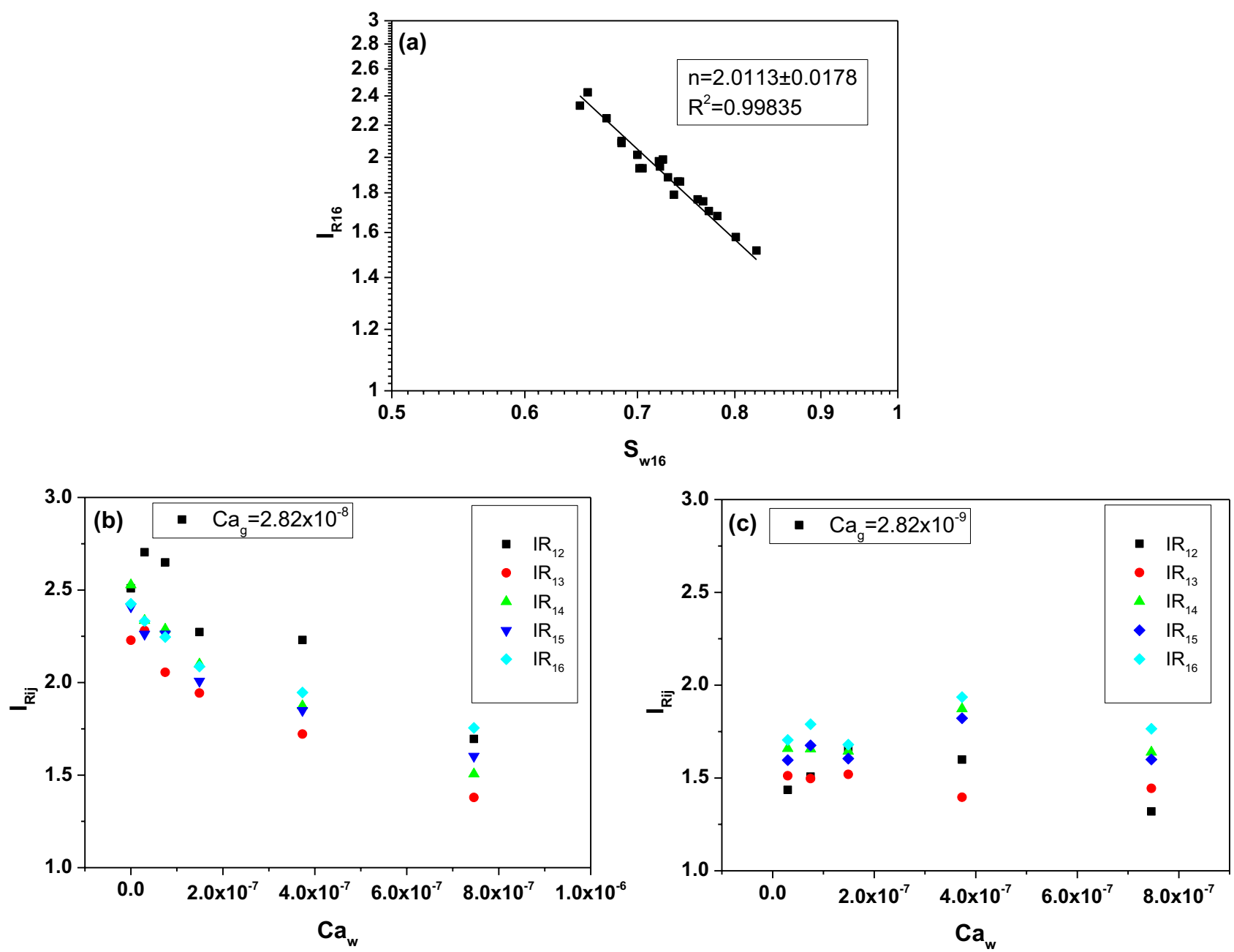

Fig. 4. (a) Calibration of steady-state values of electrical resistivity vs. water saturation measurements. Measured steady-state resistivity index across cumulative segments of the sand column as a function of water capillary number for $(\mathrm{b}) \mathrm{Ca}_{\mathrm{g}}=2.82 \times 10^{-8}$ and (c) $\mathrm{Ca}_{\mathrm{g}}=2.82 \times 10^{-9}$.

values of $\kappa$, the non-wetting fluid is mainly disconnected into ganglia moving and undergoing coalescence, break-up, fission and trapping, so that the flow regime is dominated exclusively by various types of oil Ganglion Dynamics (GD): Large Ganglion Dynamics (LGD), Small Ganglion Dynamics (SGD), Drop Traffic Flow (DTF) (Avraam and Payatakes, 1995a). In the present case of a gas/water system with a low viscosity ratio, $\kappa=\mu_{\mathrm{g}} / \mu_{\mathrm{w}}=0.02$, and high values of water saturation, even at relatively low $\mathrm{Ca}_{\mathrm{w}}$ values (Fig. 6), no connected pathway flow of gas phase is expected, unless the gas flow rate becomes so high that the Darcy's law ceases. Therefore, the gas phase flow regime is expected to be dominated by GD over the full range of $\mathrm{Ca}_{\mathrm{w}}$ and $\mathrm{Ca}_{\mathrm{g}}$ values, examined.

With the aid of mass balances, values of the resistivity index measured over cumulative segments of the column (Figs. 4b and 4c) were converted to water saturation values, $\left\langle S_{\mathrm{w}}\right\rangle_{i j}(i j=12,23,34,45,56)$, averaged over successive segments of the column. These saturations are plotted as function of the dimensionless axial distance of the middle of each segment from the column inlet, $\left(\xi_{\mathrm{m}}\right)_{i j}\left(\xi_{\mathrm{m}}=x_{\mathrm{m}} / L\right)$ (Fig. 6).
Evidently the water saturation averaged over each of the five successive segments of the column, $\left\langle S_{\mathrm{w}}\right\rangle_{i j}(i j=12,23,34,45$, 56 ) varies noticeably (Fig. 6 ) and this might be attributed to the non-fully developed two-phase flow at the vicinity of inlet and outlet ports, and the capillary end effect (Aggelopoulos and Tsakiroglou, 2008). When both fluids are injected simultaneously, the irreducible water saturation, trapped in the porous medium at the end of displacement, reconnects with the bulk phase so that water saturation increases rapidly in the first segments of the column (Fig. 6a).

\subsection{Dependence of relative permeabilities on capillary numbers}

With the aid of Athena Visual Studio (Stewart and Caracotsios, 2008), the experimental results were fitted to equations (4)-(6) to estimate all unknown parameters (Tabs. 2 and 3). Assuming that the water saturation averaged over each segment follows the power law

$$
\left\langle S_{\mathrm{w}}\right\rangle_{i j}=\left(a_{\mathrm{s}}\right)_{i j} \mathrm{Ca}_{\mathrm{w}}{ }^{{ }_{\mathrm{w}}} \mathrm{Ca}_{\mathrm{g}}{ }^{{ }^{\mathrm{g}}},
$$



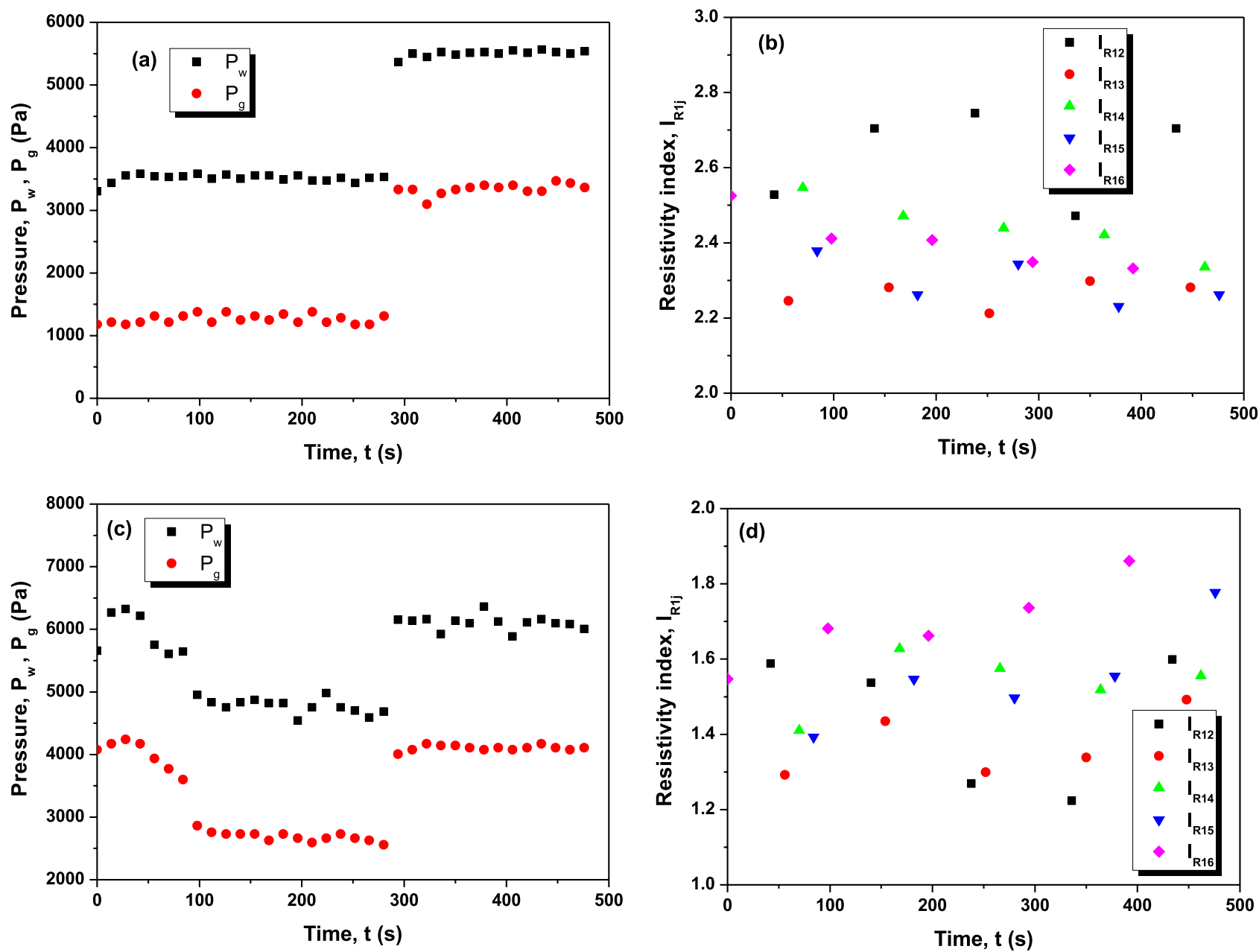

Fig. 5. Transient responses of the (a, c) water/gas inlet pressures, (b, d) resistivity index across cumulative segments of the sand column for $(\mathrm{a}, \mathrm{b}) q_{\mathrm{w}}=0.2 \mathrm{~mL} / \mathrm{min}, q_{\mathrm{g}}=10 \mathrm{~mL} / \mathrm{min}$, and $(\mathrm{c}, \mathrm{d}) q_{\mathrm{w}}=1.0 \mathrm{~mL} / \mathrm{min}, q_{\mathrm{g}}=2.5 \mathrm{~mL} / \mathrm{min}$.

then, for fixed values of $\mathrm{Ca}_{\mathrm{w}}$ and $\mathrm{Ca}_{\mathrm{g}}$, the observed local variation of $\left\langle S_{\mathrm{w}}\right\rangle_{i j}$ across the column (Fig. 6) is reflected in the variation of the pre-exponential coefficient $\left(a_{\mathrm{s}}\right)_{i j}$ from segment to segment and might be attributed to the different boundary conditions prevailing at the ends of each segment. From the mass balance of water saturation, the column-averaged coefficient, $a_{\mathrm{s}}$, is expected to be equal to the arithmetic average of local coefficients, $\left(a_{\mathrm{s}}\right)_{i j}$, namely

$$
a_{\mathrm{s}}=\sum_{i j} \xi_{i j}\left(a_{\mathrm{s}}\right)_{i j}
$$

The experimentally measured and numerically estimated, equations (4)-(6), column-averaged relative permeability of aqueous, $\left\langle k_{\mathrm{rw}}\right\rangle$, and gas $\left\langle k_{\mathrm{rg}}\right\rangle$ phase along with the column-averaged water saturation are shown as functions of capillary numbers in Figure 7, and the estimated parameter values are given in Tables $2-4$. The water saturation datasets were fitted to equations (6) and (8) to estimate the parameters $c_{\mathrm{w}}, c_{\mathrm{o}},\left(a_{\mathrm{s}}\right)_{i j} i j=12,23,34,45,56$ and calculate the parameter $a_{\mathrm{s}}$, from equation (9) (Tab. 2).
In general, over the range of experimentally measured water saturations, $\left\langle S_{\mathrm{w}}\right\rangle(\sim 0.6-0.8),\left\langle k_{\mathrm{rw}}\right\rangle$ is low (Fig. 7) since the compact structure of wetting fluid is fragmented into moving or trapped ganglia of gas phase (bubbles), so that a significant fraction of water saturation belongs to "islands" which are immobile or move slowly and their contribution to $\left\langle k_{\mathrm{rw}}\right\rangle$ is nil. The relative permeability of aqueous phase is more sensitive to $\mathrm{Ca}_{\mathrm{w}}$ and less sensitive to $\mathrm{Ca}_{\mathrm{g}}$ (Fig. 7, Tab. 3). As $\mathrm{Ca}_{\mathrm{w}}$ increases, the aqueous phase starts occupying a gradually increasing number of relatively narrow but non-interrupting paths spanning the porous medium and "transferring" most of the flow (Tallakstad et al., 2009a) so that the hydraulic conductivity of wetting fluid or equivalently the value of $\left\langle k_{\mathrm{rw}}\right\rangle$ increases appreciably (Fig. 7). The viscous coupling between gas and aqueous phase is reflected in the weak but negative effect of $\mathrm{Ca}_{\mathrm{w}}$ on $\left\langle k_{\mathrm{rg}}\right\rangle$ (Fig. 7, Tab. 4). As mentioned earlier, the flow regime of gas phase is governed by GD, the increase of $\mathrm{Ca}_{\mathrm{w}}$ is associated with fewer ganglia of gas phase with smaller mean and cut-off sizes (Tallakstad et al., 2009a), so that a weak decrease of $\left\langle k_{\mathrm{rg}}\right\rangle$ is observed (Avraam and Payatakes, 1995a; Tsakiroglou et al., 2015). 

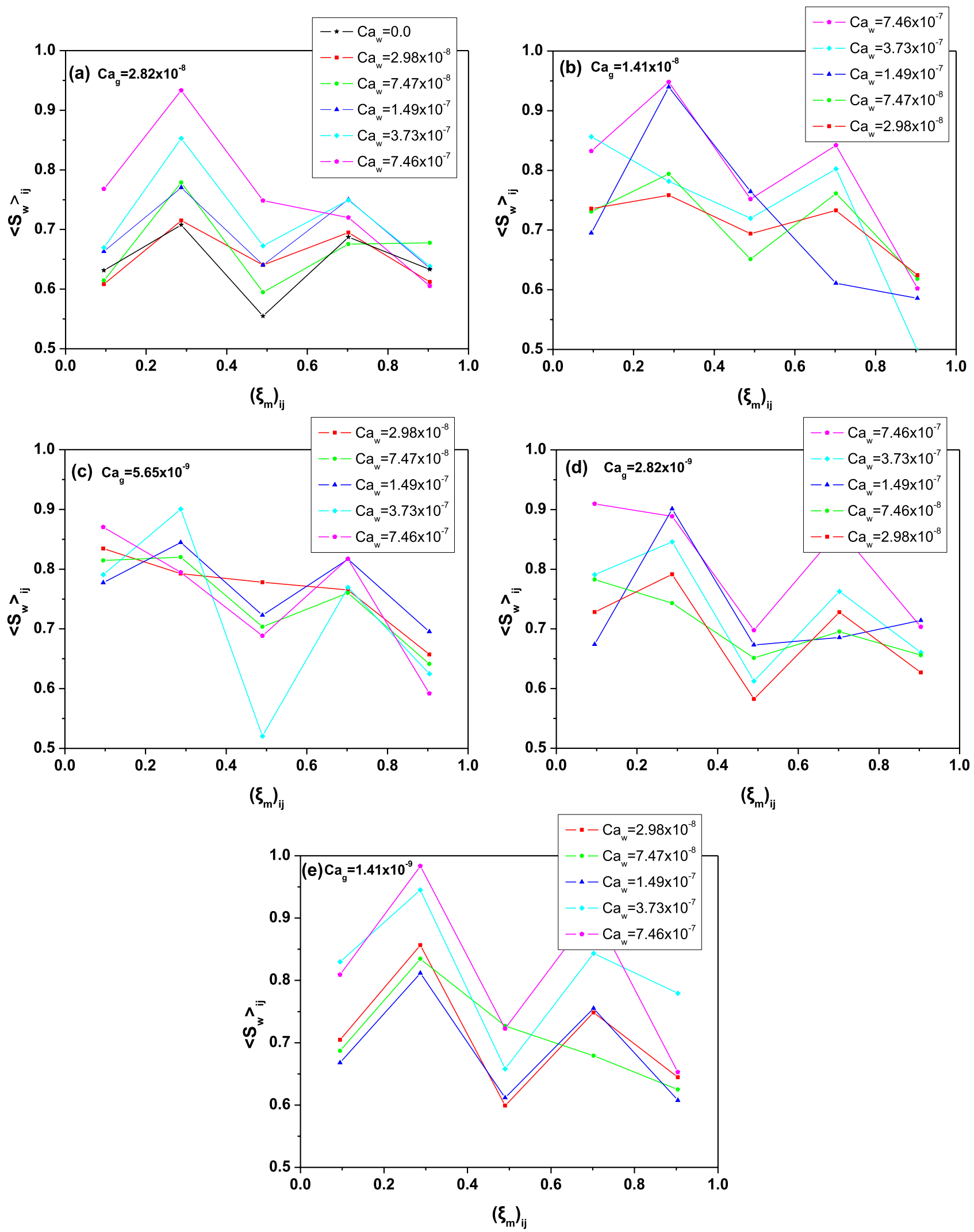

Fig. 6. Variation of the axial distribution of column segment-averaged water saturation as a function of capillary number: (a) $\mathrm{Ca}_{\mathrm{g}}=2.82 \times 10^{-8}$; (b) $\mathrm{Ca}_{\mathrm{g}}=1.41 \times 10^{-8}$; (c) $\mathrm{Ca}_{\mathrm{g}}=5.65 \times 10^{-9}$; (d) $\mathrm{Ca}_{\mathrm{g}}=2.82 \times 10^{-9}$; (e) $\mathrm{Ca}_{\mathrm{g}}=1.41 \times 10^{-9}$. 
Table 2. Estimated values of parameters relating $\left\langle S_{\mathrm{w}}\right\rangle_{i j}$ with $\mathrm{Ca}_{\mathrm{w}}$ and $\mathrm{Ca}_{\mathrm{g}}$, equation (8). All parameters are unitless.

\begin{tabular}{lc}
\hline Parameter & Mean value \pm standard deviation \\
\hline$c_{\mathrm{w}}$ & $0.0329 \pm 0.0056$ \\
$c_{\mathrm{g}}$ & $-0.01905 \pm 0.0061$ \\
$a_{\mathrm{s}}$ & 0.8573 \\
$\left(a_{\mathrm{s}}\right)_{12}$ & $0.8824 \pm 0.13$ \\
$\left(a_{\mathrm{s}}\right)_{23}$ & $0.9869 \pm 0.151$ \\
$\left(a_{\mathrm{s}}\right)_{34}$ & $0.7862 \pm 0.116$ \\
$\left(a_{\mathrm{s}}\right)_{45}$ & $0.8868 \pm 0.131$ \\
$\left(a_{\mathrm{s}}\right)_{56}$ & $0.7466 \pm 0.110$ \\
\hline
\end{tabular}

Likewise, the gas relative permeability is more sensitive to $\mathrm{Ca}_{\mathrm{g}}$ and less sensitive to $\mathrm{Ca}_{\mathrm{w}}$ (Fig. 7, Tab. 4). As $\mathrm{Ca}_{\mathrm{g}}$ increases, a progressively higher number of pore clusters occupied by trapped gas phase ganglia is mobilized, leading to a higher number of moving ganglia and resulting in a significant increase of $\left\langle k_{\mathrm{rg}}\right\rangle$ (Fig. 7). At increasing $\mathrm{Ca}_{\mathrm{g}}$ values, the size of ganglia is expected to decrease (Avraam and Payatakes, 1995a). On the other hand, the $\left\langle k_{\mathrm{rw}}\right\rangle$ is affected weakly by $\mathrm{Ca}_{\mathrm{g}}$ since water retains its hydraulic continuity over the full range of $\mathrm{Ca}_{\mathrm{g}}$ values, through connected pathways and thin films or pendular rings surrounding the solid grains. The increased population of gas-phase ganglia, caused by the increase of $\mathrm{Ca}_{\mathrm{g}}$ leads to a relatively limited reduction of the size of "red" bonds, with the highest contribution fraction to the wetting fluid flow, and therefore to a weak reduction of $\left\langle k_{\mathrm{rw}}\right\rangle$ (Fig. 7).

The results of the present study along with corresponding ones from earlier studies concerning oil/water fluid systems tested in a 3-D sand column (Tsakiroglou et al., 2015) and a 2-D glass-etched pore network (Avraam and Payatakes, 1995a) are summarized in Table 5, in terms of the viscosity ratio of non-wetting $(\mathrm{nw}=\mathrm{o}, \mathrm{g})$ to wetting (w) fluid. It is worth mentioning that: (a) each relative permeability is affected by both $\mathrm{Ca}_{\mathrm{w}}$ and $\mathrm{Ca}_{\mathrm{nw}}$ (Tab. 5); (b) the exponents $b_{\mathrm{w}}$ and $e_{\mathrm{nw}}$ are higher in a 3-D rather than a 2-D pore system; (c) due to the small hydraulic resistance of non-wetting phase (gas), the dependence of water saturation on $\mathrm{Ca}_{\mathrm{w}}$ and $\mathrm{Ca}_{\text {nw }}$ becomes very weak (namely the values of $c_{\mathrm{w}}, c_{\mathrm{nw}}$ tend to zero), for a 3-D system and low viscosity ratio (Tab. 5); (d) regarding the non-wetting fluid relative permeability (Fig. 8), as the viscosity ratio decreases, the wetting fluid is facilitated to occupy connected flow pathways, the fraction of non-wetting fluid belonging to trapped ganglia is enhanced, and the slope, $\lambda_{\text {nw }}$, decreases, for both 3 -D and 2-D pore systems (Tab. 5); (e) particularly with reference to the wetting phase relative permeability (Fig. 8), the slope, $\lambda_{\mathrm{w}}$, for the gas/water system is much higher than the corresponding one estimated from tests of an oil/water system (viscosity ratio $\kappa=1.0$ ) on the same porous medium (Tab. 5). The low hydraulic conductance of the wetting fluid is due mainly to the presence of abundant trapped ganglia of gas (bubbles) or oil in the pore network. In a gas/water
Table 3. Estimated values of parameters relating $\left\langle k_{\mathrm{rw}}\right\rangle$ with $\mathrm{Ca}_{\mathrm{w}}$ and $\mathrm{Ca}_{\mathrm{g}}$, equation (4). All parameters are unitless.

\begin{tabular}{lc}
\hline Parameter & Mean value \pm standard deviation \\
\hline$a_{\mathrm{w}}$ & $13786.6 \pm 32500$ \\
$b_{\mathrm{w}}$ & $1.01205 \pm 0.1425$ \\
$b_{\mathrm{g}}$ & $-0.144 \pm 0.0584$ \\
\hline
\end{tabular}

system, with the $\mathrm{Ca}_{\mathrm{w}}$ increasing, due to the negligible viscous resistance of gas phase, the mobilization of gas bubbles is facilitated even for a small increase of water saturation so that the water conductance might increase respectably. In contrast, in an oil/water system, a fraction of the momentum earned with the $\mathrm{Ca}_{\mathrm{w}}$ increasing, is dissipated in configurational changes of trapped oil phase, making more difficult the mobilization of oil ganglia, and leading to moderate increase of water conductance.

\subsection{Impact on core analysis and reservoir engineering}

Commonly, the steady-state relative permeabilities of reservoir rocks are measured on a core plug, with well-known characteristics of porosity, by co-injecting at varying values of flow rates, a wetting, w, and a non-wetting, nw, fluid with well-known physicochemical properties $\left(\mu_{\mathrm{w}}\right.$, $\left.\mu_{\mathrm{nw}}, \gamma_{\mathrm{wnw}}, \theta_{\mathrm{e}}\right)$. The measured relative permeabilities are simply expressed as functions of wetting fluid saturation. However, the present work along with earlier ones (Avraam and Payatakes, 1995a, 1995b, 1999; Tallakstad et al., 2009a, 2009b; Tsakiroglou et al., 2007, 2015) reveals that both the wetting fluid, $k_{\mathrm{rw}}$, and non-wetting fluid, $k_{\mathrm{rnw}}$, relative permeability along with the wetting fluid saturation, $S_{\mathrm{w}}$, are governed by the wetting fluid, $\mathrm{Ca}_{\mathrm{w}}$, and nonwetting fluid, $\mathrm{Ca}_{\mathrm{nw}}$, capillary number, namely

$$
\begin{gathered}
k_{\mathrm{rw}}=G_{\mathrm{w}}\left(\mathrm{Ca}_{\mathrm{w}}, \mathrm{Ca}_{\mathrm{nw}}\right), \\
k_{\mathrm{rnw}}=G_{\mathrm{nw}}\left(\mathrm{Ca}_{\mathrm{w}}, \mathrm{Ca}_{\mathrm{nw}}\right), \\
S_{\mathrm{w}}=H_{\mathrm{w}}\left(\mathrm{Ca}_{\mathrm{w}}, \mathrm{Ca}_{\mathrm{nw}}\right) .
\end{gathered}
$$

Therefore, a new formulation of the two-phase flow equations is required where $k_{\mathrm{rw}}, k_{\mathrm{rnw}}, S_{\mathrm{w}}$, are handled as dependent variables and $\mathrm{Ca}_{\mathrm{w}}, \mathrm{Ca}_{\mathrm{nw}}$, as independent variables.

In addition, earlier studies have shown that the dynamic capillary pressure curve measured by rate-controlled immiscible displacement tests depends also on the flow rates (Tsakiroglou et al., 2003; Aggelopoulos and Tsakiroglou, 2008; Joekar-Niasar et al., 2010). Subsequently, a new generalized numerical approach of the transient two-phase flow in porous media might be on $\mathrm{Ca}_{w^{-}}, \mathrm{Ca}_{\mathrm{nw}^{-}}$dependent two-phase flow functions $\left(k_{\mathrm{rw}}, k_{\mathrm{rnw}}, S_{\mathrm{w}}, P_{\mathrm{c}}\right)$. From this point of view, it might be necessary to mention not only the wetting fluid saturation but also the $\mathrm{Ca}_{\mathrm{w}}, \mathrm{Ca}_{\mathrm{nw}}$ values, when conducting steady-state 2-phase flow tests on cores of reservoir rocks. 

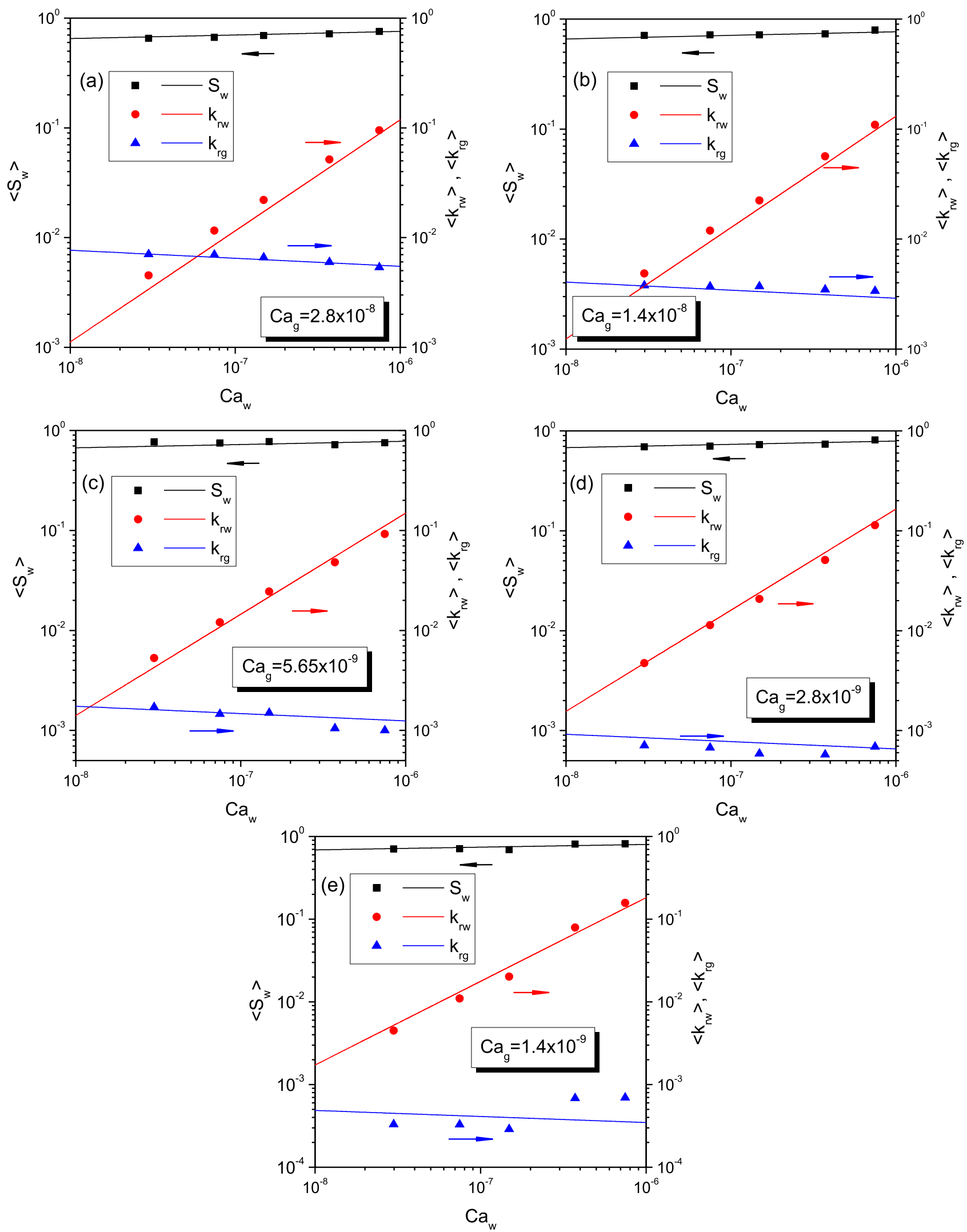

Fig. 7. Column-averaged water saturation and water/gas relative permeability as functions of $\mathrm{Ca}_{\mathrm{w}}$ for various values of $\mathrm{Ca}$. The coloured arrows indicate the vertical axis reference (saturation or relative permeability) for the corresponding lines. 
Table 4. Estimated values of parameters relating $\left\langle k_{\mathrm{rg}}\right\rangle$ with $\mathrm{Ca}_{\mathrm{w}}$ and $\mathrm{Ca}_{\mathrm{g}}$, equation (5). All parameters are unitless.

\begin{tabular}{lc}
\hline Parameter & Mean value \pm standard deviation \\
\hline$a_{\mathrm{g}}$ & $21714.6 \pm 24170$ \\
$e_{\mathrm{w}}$ & $-0.0732 \pm 0.0244$ \\
$e_{\mathrm{g}}$ & $0.9217 \pm 0.0585$ \\
\hline
\end{tabular}

Table 5. Summarized results of the exponents estimated from tests in 3-D and 2-D pore systems.

\begin{tabular}{lcccc}
\hline Network & $3-\mathrm{D}$ & $3-\mathrm{D}$ & $2-\mathrm{D}$ & $2-\mathrm{D}$ \\
\hline$\kappa$ & 0.02 & 1.0 & 1.45 & 3.35 \\
$c_{\mathrm{w}}$ & 0.0329 & 0.1321 & 0.132 & 0.144 \\
$c_{\mathrm{nw}}$ & -0.019 & -0.226 & -0.10 & -0.112 \\
$b_{\mathrm{w}}$ & 1.012 & 0.8845 & 0.646 & 0.583 \\
$b_{\mathrm{nw}}$ & -0.144 & -0.255 & -0.178 & -0.187 \\
$e_{\mathrm{w}}$ & -0.073 & -0.167 & -0.197 & -0.09 \\
$e_{\mathrm{nw}}$ & 0.9217 & 0.720 & 0.6405 & 0.639 \\
$\lambda_{\mathrm{w}}=b_{\mathrm{w}} / c_{\mathrm{w}}$ & 30.76 & 6.695 & 4.894 & 4.049 \\
$\lambda_{\mathrm{nw}}=e_{\mathrm{w}} / c_{\mathrm{w}}$ & -2.219 & -1.264 & -1.492 & -0.625 \\
\hline
\end{tabular}

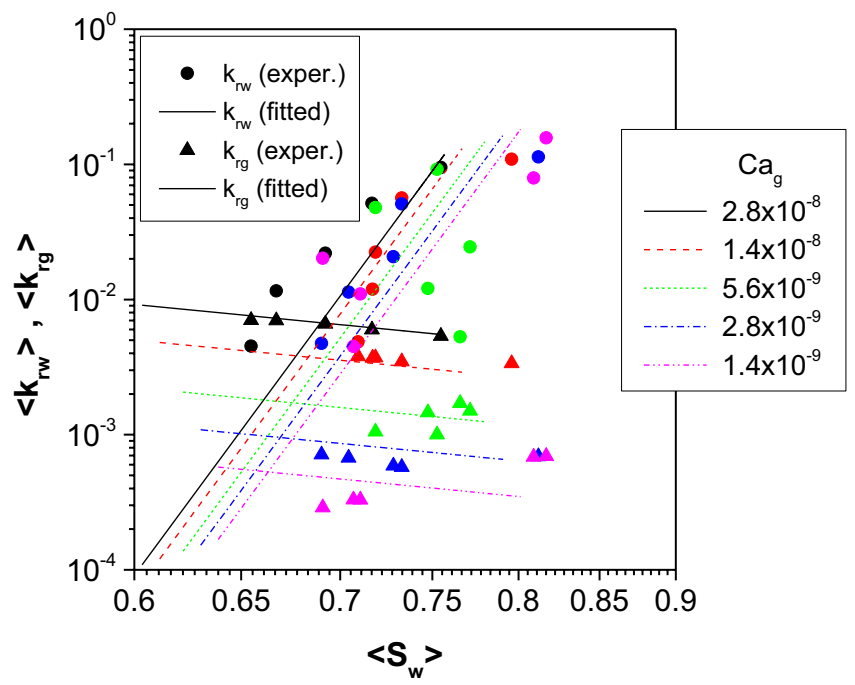

Fig. 8. Steady-state gas/water relative permeability functions for the sand column.

\section{Conclusion}

Experiments of the steady-state $\mathrm{N}_{2}$ /brine flow in a sand column, for varying values of gas and brine capillary numbers, are analyzed in the framework of rate-dependent relative permeability functions. The pressure drop across each phase is measured with differential pressure transducers. The axial profile of brine saturation is measured by recording the transient response of the resistivity index across five segments of the porous medium with an in-house constructed multi-point electrical conductivity meter. The effect of the gas and water capillary numbers on both gas and water relative permeabilities is quantified by estimating the relevant exponents of power laws, and the results are summarized along with those of earlier studies for oil/water fluid systems. The main conclusions are outlined below:

- In agreement with earlier studies, it seems that water is flowing in the form of connected pathways and disconnected ganglia, while the gas flow regime is dominated by disconnected ganglia (bubbles).

- The water saturation averaged over the sand column changes weakly with the water and gas capillary numbers.

- Each relative permeability is affected by both water and gas capillary number, the water relative permeability is a strong function of water capillary number, and the gas relative permeability changes strongly with the gas capillary number.

- The slope of the water relative permeability curve for a gas/water system is much higher than that of an oil/ water system, and the slope of the gas relative permeability is lower than that of an oil/water system.

\section{References}

Aggelopoulos C., Klepetsanis P., Theodoropoulou M., Pomoni K., Tsakiroglou C.D. (2005) Large-scale effects on the resistivity index of porous media, J. Contam. Hydrol. 77, 299-323.

Aggelopoulos C.A., Tsakiroglou C.D. (2008) The effect of microheterogeneity and capillary number on capillary pressure and relative permeability curves of soils, Geoderma 148, 25-34.

Avraam D.G., Payatakes A.C. (1995a) Flow regimes and relative permeabilities during steady-state two-phase flow in porous media, J. Fluid Mech. 293, 207-236.

Avraam D.G., Payatakes A.C. (1995b) Generalized relative permeability coefficients during steady-state two-phase flow in porous media and correlation with the flow mechanisms, Transp. Porous Media 20, 135-168.

Avraam D.G., Payatakes A.C. (1999) Flow mechanisms, relative permeabilities, and coupling effects in steady-state two-phase flow through porous media. The case of strong wettability, Ind. Eng. Chem. Res. 38, 778-786.

Constantinides G.N., Payatakes A.C. (1996) Network simulation of steady-state two-phase flow in consolidated porous media, AIChE J. 42, 369-382.

Eftekhari A.A., Farajzadeh R. (2017) Effect of foam on liquid phase mobility in porous media, Scientific Reports 7, 43870.

Erpelding M., Sinha S., Tallakstad K.T., Hansen A., Flekkoy E. G., Maloy K.J. (2013) History independence of steady-state in simultaneous two-phase flow through two-dimensional porous media, Phys. Rev. E 88, 053004.

Grøva M., Hansen A. (2011) Two-phase flow in porous media: power-law scaling of effective permeability, J. Phys. Conf. Ser. 319, 012009.

Gutierrez B., Juarez F., Ornelas L., Zeppieri S., Lopez de Ramos A. (2008) Experimental study of gas-liquid two-phase flow in glass micromodels, Int. J. Thermophys. 29, 2126-2135. 
Joekar-Niasar V., Hassanizadeh M., Dahle H.K. (2010) Non-equilibrium effects in capillarity and interfacial area in two-phase flow: dynamic pore-network modelling, J. Fluid Mech. 655, 38-71.

Kamali F., Hussain F. (2017) Field-scale simulation of $\mathrm{CO}_{2}$ enhanced oil recovery and storage through SWAG injection using laboratory estimated relative permeabilities, J. Pet. Sci. Eng. 156, 396-407.

Johnson A., Patil S., Dandekar A. (2011) Experimental investigation of gas-water relative permeability for gas-hydrate bearing sediments from the Mount Elbert Gas Hydrate Stratigraphic Test Well Alaska North Slope, Marine Petrol. Geol. 28, 419-426.

Ramstad T., Hansen A.H. (2006) Cluster evolution in steadystate two-phase flow in porous media, Phys. Rev. E 73, 026306.

Ramstad T., Idowu N., Nardi C., Oren P.-E. (2012) Relative permeability calculations from two-phase flow simulations directly on digital images of porous rocks, Transp. Porous Media 94, 487-504.

Reynolds C.A., Krevor S. (2015) Characterizing flow behavior for gas injection: Relative permeability of $\mathrm{CO}_{2}$-brine and $\mathrm{N}_{2}$-water in heterogeneous rocks, Water Resour. Res. WR018046, 9464-9489.

Sherafati M., Jessen K. (2017) Dynamic relative permeability and Simulation of WAG injection processes, Transp. Porous Media 117, 125-147.

Sidiq H., Amin B., Kennaird T. (2017) The study of relative permeability and residual gas saturation at high pressures and high temperatures, Adv. Geoenergy Res. 1, 64-68.

Sinha S., Hansen A. (2012) Effective rheology of immiscible twophase flow in porous media, Europhys. Lett. 99, 44004.

Stewart W.E., Caracotsios M. (2008) Computer-Aided Modeling of Reactive Systems, John Wiley \& Sons, Hoboken, New Jersey.

Tallakstad K.T., Lovoll G., Knudsen H.A., Ramstad T., Flekkoy E.G., Maloy K.J. (2009a) Steady-state, simultaneous twophase flow in porous media: an experimental study, Phys. Rev. E 80, 036308 .

Tallakstad K.T., Knudsen H.A., Ramstad T., Lovoll G., Maloy K.J., Toussaint R., Flekkoy E.G. (2009b) Steady-state two-phase flow in porous media: statistics and transport properties, Phys. Rev. Lett. 102, 074502.

Terzi K., Bountas I., Aggelopoulos C.A., Tsakiroglou C.D. (2014) Effects of carbon dioxide on the mobilization of metals from aquifers, Environ. Sci. Technol. 148, 4386-4394.

Tsakiroglou C.D., Avraam D.G., Payatakes A.C. (2007) Transient and steady-state relative permeabilities from two-phase flow experiments in planar pore networks, Adv. Water Res. 30, 1981-1992.

Tsakiroglou C.D., Aggelopoulos C.A., Terzi K., Avraam D.G., Valavanides M. (2015) Steady-state two-phase relative permeability functions of porous media: a revisit, Int. J. Multiphase Flow 73, 34-42.

Tsakiroglou C.D., Theodoropoulou M., Karoutsos V. (2003) Non-equilibrium capillary pressure and relative permeability curves of porous media, AIChE J. 49, 2472-2486.

Valavanides M.S., Constantinides G.N., Payatakes A.C. (1998) Mechanistic model of steady-state two-phase flow in porous media based on ganglion dynamics, Transp. Porous Media $\mathbf{3 0}$, 267-299.

Valavanides M.S. (2012) Steady-state two-phase flow in porous media: Review of progress in the development of the DeProF theory bridging pore to statistical thermodynamics scales, $O i l$ Gas Sci. Technol. - Rev. IFP Energies nouvelles 67, 787-804.

Valavanides M.S., Totaj E., Tsokopoulos M. (2016) Energy efficiency characteristics in steady-state relative permeability diagrams of two-phase flow in porous media, J. Petrol. Sci. Eng. 147, 181-201.

Wang Y., Bryan C., Dewers T., Heath J.E., Jove-Colon C. (2013) Ganglion dynamics and its implications to geologic carbon dioxide storage, Environ. Sci. Technol. 47, 219-226.

Wu F., Fan Q., Huang D., Ma L., Linag X., Sima L. (2016) Predicting gas-water relative permeability using nuclear magnetic resonance and mercury injection capillary pressure measurements, J. Nat. Gas Sci. Eng. 32, 35-47.

Zhang D., Papadikis K., Gu S. (2016) A lattice Boltzmann study on the impact of the geometrical properties of porous media on the steady state relative permeabilities on two-phase immiscible flows, Adv. Water Resour. 95, 61-79. 